\title{
RELIABILITY TESTS FOR DOPPLER IMAGING
}

\author{
Y.C. UNRUH \\ University of St Andrews \\ Dept of Physics $\mathcal{E}$ Astronomy, North Haugh, St Andrews, Fife, \\ KY16 $9 S S$
}

\begin{abstract}
In recent years, Doppler imaging codes have been developed by a number of different groups and successfully applied to map the spot distribution on stellar surfaces. As more and more images are obtained we tend to see a pattern of high-latitude spots, particularly on RSCVn systems. These high-latitude spots were and still are greeted with scepticism as they are inferred from non-varying profile deformations. Here, I describe some of the problems of Doppler imaging and their likely impact on the resulting images.
\end{abstract}

\section{Introduction}

The technique of Doppler imaging and different implementations have been described in a number of papers (Vogt \& Penrod, 1983; Rice, Wehlau \& Khokhlova, 1989; Piskunov, 1990; Collier Cameron, 1992; Kürster, 1993). The results that are presented here are based on tests performed with a Doppler imaging code written by A. Collier Cameron (Collier Cameron, 1992; Collier Cameron \& Unruh, 1994). Its main particularity is that it uses a two-temperature approach whereby each pixel on the stellar surface is assigned a 'spot filling factor' describing the percentage of the pixel surface covered by cool spots.

In order to map the surface of a star we need to know some of its basic parameters, notably its spectral type, rotation period, rotation velocity and inclination angle. Most of these parameters will not be known exactly and hence introduce errors in the reconstructions.

We also need to know the shape of the unbroadened line profile of the particular mapping line as a function of temperature and limb angle, so 
that we can compare the stellar data to the model that we derive from our inferred surface distribution. Again, the shape of this 'intrinsic' line profile is not certain and we should investigate how errors in the intrinsic shape will propagate into the final reconstruction.

Furthermore, we have to cope with all the 'real-world' effects, such as a final signal-to-noise level, limited spectral resolution, the fact that most lines are blended and that we usually have only limited phase coverage.

\section{Errors in the stellar parameters}

One of the easiest way of testing the sensitivity of Doppler imaging reconstructions is to create synthetic data from a given image, introduce some noise and reconstruct an image from the synthetic data with changed parameters. The reconstructed image can then be compared with the original image to check how well the different features have been reconstructed and where artefacts have arisen. Most investigators have checked the sensitivity of their codes with respect to errors in the rotation velocity and the inclination angle (see for example Vogt, Penrod \& Hatzes (1987) and Kürster (1993)). Errors associated with the intrinsic line shape have been less well investigated (Unruh \& Collier Cameron, 1995; Piskunov \& Rice, 1993).

\subsection{ERRORS IN THE PROJECTED ROTATION VELOCITY}

Doppler images are very sensitive to errors in the projected rotation velocity, $v \sin i$. For large errors (more than about 10 per cent when the velocity is overestimated, but only of the order of 5 per cent when the velocity is underestimated), it is usually not possible to find a converging solution. For less drastic errors, the resulting images show artificial banding. If the value of $v \sin i$ is overestimated, the artificial band will lie close to the equator; for underestimated $v \sin i$ values we will see a polar spot.

\subsection{INCLINATION ANGLE}

Doppler imaging is also sensitive to the stellar inclination, though small errors of the order of $\pm 10^{\circ}$ or so will still yield very satisfactory reconstructions. If the inclination angle is above about $60^{\circ}$ and we overestimate it, the latitudinal smearing and mirroring between the northern and southern hemisphere increases. An artificial polar spot can appear for lower inclination angles. If the inclination angle is underestimated, the existing structure gets compressed in latitude and a band at mid-latitude appears.

Note that artificial banding might not always appear in the form of a smooth homogeneous band. For data with poor signal-to-noise ratio, one 


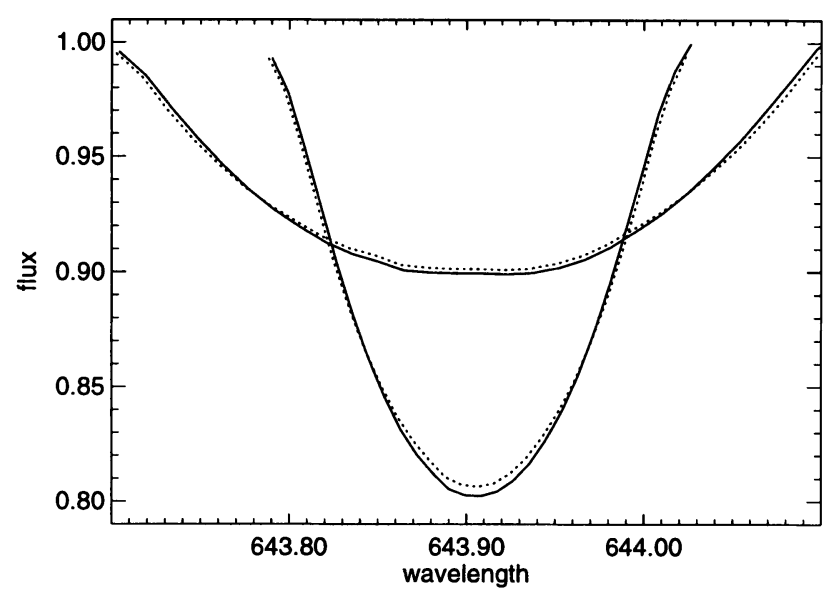

Figure 1. Rotationally broadened profiles, $v \sin i=50 \mathrm{~km} \mathrm{~s}^{-1}$ (deep curves) and $v \sin i=90 \mathrm{~km} \mathrm{~s}^{-1}$ (shallow curves), comparing the constant-profile approximation to a full spectral synthesis calculation for the $\mathrm{Ca}$ I line at $643.9 \mathrm{~nm}$ and $T=5000 \mathrm{~K}$. The solid lines show the rotationally broadened profiles for the spectral synthesis calculation, the dotted lines represent the constant-profile approach. The equivalent width of the constant profile corresponds to the equivalent width of disk-integrated variable profile.

finds that noise features will be 'fitted' and pushed towards a preferred latitude. Similarly, real features will tend to be pulled towards that latitude.

\section{Errors in the line profile}

\subsection{THE SHAPE OF THE LINE PROFILE AS A FUNCTION OF LIMB ANGLE}

When solving the radiative transfer equation for different limb angles, one finds that the profile shape is a function of the position on the star. Usually, the intensity minimum at the line centre changes very little as one moves from the centre towards the limb, whereas the continuum intensity decreases strongly. If we compare the intensity profiles with the diskintegrated profile that has been scaled to fit a linear limb darkening law, we find a relative decrease in the profile depth for increasing limb angles.

Not all codes include this variation of the profile shape and its neglect can often be justified on the grounds that the resulting rotationally broadened profile of a constant line looks very similar to the profile that is obtained by broadening a varying profile. The difference between the two profiles is shown in Fig. 1 for two different values of $v \sin i$. It shows that the neglect of the line profile variation as a function of limb angle results in a flatter rotationally broadened profile. Fig. 2(c) shows an example 
where data produced with a variable profile is reconstructed with a constant profile, resulting in an image with a spurious band at low latitudes. This result seems to contradict the findings by Piskunov \& Rice (1993). For $\mathrm{Ca} I$ at $643.9 \mathrm{~nm}$ and other lines where the equivalent width increases strongly with decreasing temperature, they found a weak temperature gradient with slightly cooler material towards the pole when the line profiles variation was neglected. These two apparently conflicting findings can be reconciled when one looks at how the codes can achieve a particular profile shape. If temperature is used as an image parameter, the profile of a temperature-sensitive line can be deepened by lowering the temperature. A flatter profile can hence be 'offset' by introducing a gentle temperature gradient. When the filling factor is used as image parameter, a similar effect is achieved by decreasing the amount of structure present at the pole and increasing or strengthening the structure at the equator.

The rotationally broadened lines resulting from a constant profile can be made to approach those from a full synthetic profile by a combination of two methods. We can decrease the $v \sin i$ value slightly (by about $0.5 \mathrm{~km}$ $\mathrm{s}^{-1}$ or so) and we can also decrease the equivalent width of the constant line profile (by about 1 per cent). This still leads to a slight mismatch in the line wings, though the image is recovered well (see Fig. 2(d)). As it is in practice very difficult to determine the rotation velocity or the equivalent width to better than 1 per cent, the neglect of the profile variation will be a relatively minor effect.

The change in the profile shape as a function of limb angle can be even more dramatic if one assumes an anisotropic distribution of the turbulent velocity (Unruh \& Collier Cameron, 1995). For the following tests, however, we neglect the variation of the line profile as a function of limb angle and compare different constant-profile approaches.

\subsection{MISMATCH OF THE PROFILE EQUIVALENT WIDTH}

As the equivalent width of the mapping line becomes larger than the original line width that was used to generate the data, a polar spot or high-latitude band will appear on the reconstructed image. Conversely, as the equivalent width becomes smaller, any high-latitude structure will be suppressed and a low-latitude band will show on the stellar surface. Together with $v \sin i$, the equivalent width can be 'tuned' to create or offset most 'symmetrical' misfits.

\subsection{CONTINUUM LIMB DARKENING}

The effects of misjudging the continuum limb darkening have been investigated by Kürster (1993) and Unruh \& Collier Cameron (1995). In both 

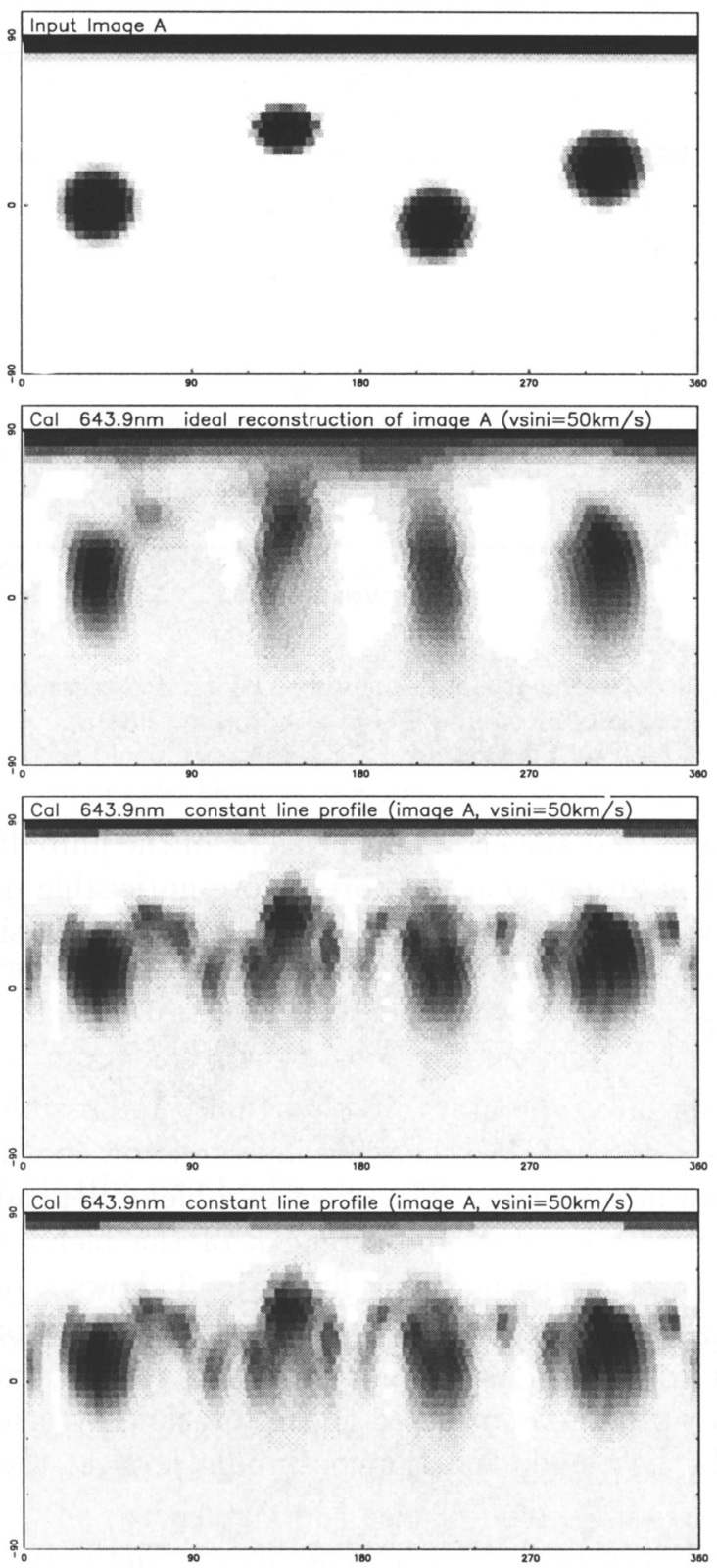

Figure 2. Artefacts due to neglecting the variability of the profile shape as a function of limb angle. The data set was produced with $\mathrm{Ca} I$ at $\lambda=643.9 \mathrm{~nm}, \mathrm{~T}=5000 \mathrm{~K}$, $v \sin i=50 \mathrm{~km} \mathrm{~s}^{-1}$ and $\mathrm{S} / \mathrm{N}$ of 340 in each velocity bin $\left(7.5 \mathrm{~km} \mathrm{~s}^{-1}\right)$. The values in brackets give the fraction of the stellar surface covered by spots. (a) Input image A (0.067); (b) 'ideal' reconstruction (0.063) taking account of the profile variation; (c) reconstruction when the profile variation is neglected and the stellar parameters are fixed $(0.086)$, and (d) similar to (c) but with a reduced rotation velocity of $49.6 \mathrm{~km} \mathrm{~s}^{-1}$ and a 1 per cent increase of the line-profile equivalent width (0.067). The differences between the constant and varying profiles become less important with increasing rotation velocity. 


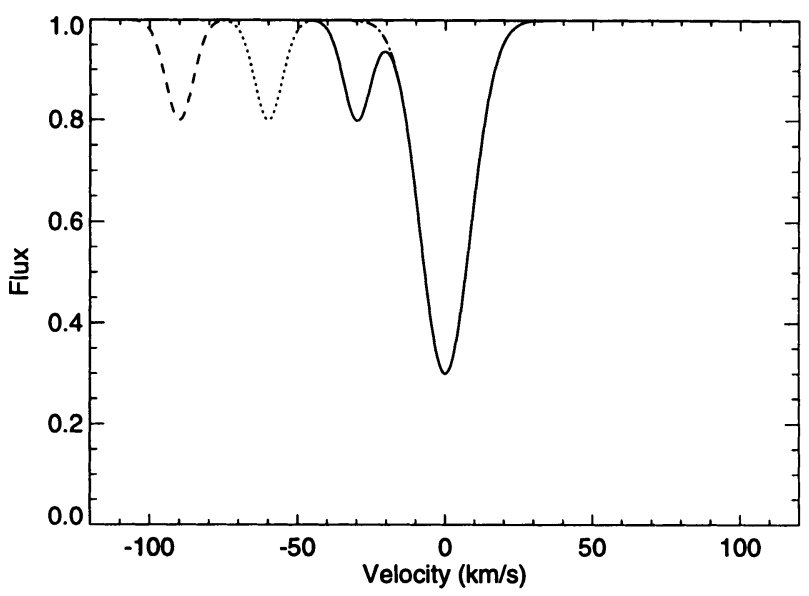

Figure 3. Examples of blended Gaussian profiles. The blends are at $-30 \mathrm{~km} \mathrm{~s}^{-1}$ (solid line), $-60 \mathrm{~km} \mathrm{~s}^{-1}$ (dotted line) and $-90 \mathrm{~km} \mathrm{~s}^{-1}$ (dashed line).

cases it was found that the errors in the slope of the limb darkening have to be of the order of 20 per cent or more before noticeable differences occur, provided that we allow some flexibility for the value of $v \sin i$.

\subsection{NEGLECTING BLENDED LINES}

For fast rotating late-type stars, it is virtually impossible to find an unblended photospheric line. We therefore have to know to what extent blends need to be taken into account. The effect of a blend depends mainly on the rotation velocity of the star, the strength of the blend and its distance from the line centre of the mapping line. Fig. 3 shows a series of test profiles with Gaussian blends and Fig. 4 shows the same profiles 'spun-up' to $v \sin i=90 \mathrm{~km} \mathrm{~s}^{-1}$. As the distance of the blend from the mapping line centre increases, the line centre of the rotationally broadened profile is shifted towards the blend, and the continuum level at the blended side is lowered.

The blended lines were used to generate synthetic data for a star with $v \sin i=90 \mathrm{~km} \mathrm{~s}^{-1}$, an inclination of $60^{\circ}$ and input image B (Fig. 5a). Synthetic data with blends closer than about $\pm 40 \mathrm{~km} \mathrm{~s}^{-1}$, could be fitted satisfactorily with a single Gaussian. For blends further out, the fits were less good and higher values of $\chi^{2}$ (the goodness of fit) had to be adopted. The reconstructed images are shown in Fig. 5 and show that, with single Gaussians, particular latitudes are emphasised on the star depending on the distance of the blend. All images were obtained by fitting the data with 


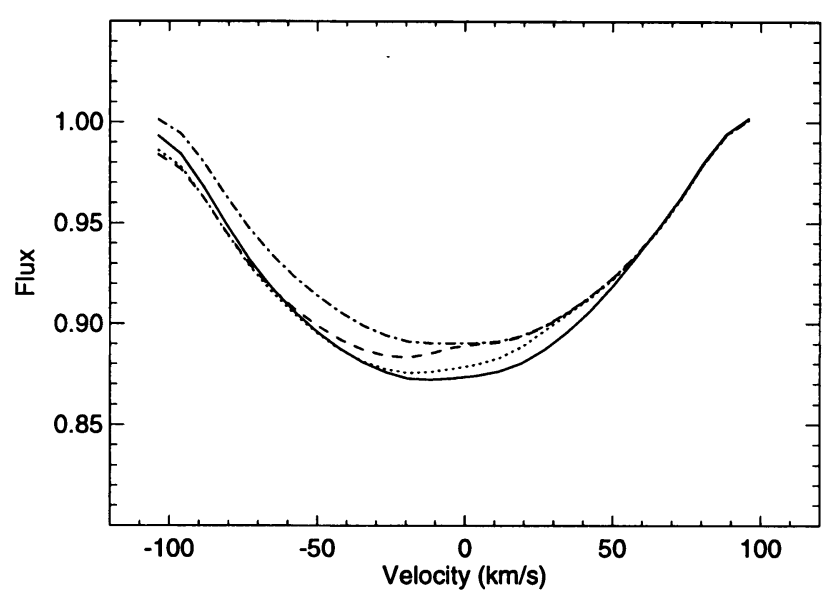

Figure 4. The rotationally broadened Gaussian profiles from Fig. 3. The rotation velocity was assumed to be $90 \mathrm{~km} \mathrm{~s}^{-1}$. As in Fig. 3, the dot-dashed line marks a single Gaussian. The solid line marks the blend at $-30 \mathrm{~km} \mathrm{~s}^{-1}$, the dotted line the blend at $-60 \mathrm{~km} \mathrm{~s}^{-1}$ and the dashed line denotes the blend at $-90 \mathrm{~km} \mathrm{~s}^{-1}$.

a series of different values for the geocentric velocity, until a minimal spot coverage was reached. Note that the blend at $-90 \mathrm{~km} \mathrm{~s}^{-1}$ that intuitively might seem least important gives a very marked effect.

\subsection{CHROMOSPHERIC EFFECTS}

It is rather difficult to simulate non-LTE effects that might cause some kind of 'filling' of the line profile. It may be possible to compare the effect of the core emission to the effect that would be produced if one had two equally strong close blends. We have produced artificial data from two very close Gaussians and found that, in a sufficiently fast rotator $(v \sin i \geq$ $50 \mathrm{~km} \mathrm{~s}^{-1}$ or so), the reconstructions using single Gaussians reproduce the input image to almost the same level as the 'ideal' reconstruction. This test assumes that the line is still very narrow at chromospheric level, which might be a rather crude assumption. Nevertheless, our tests suggest that chromospheric emission is not responsible for polar spots as long as the equivalent width of the line is set correctly. Where one fears chromospheric pollution of the mapping line, it is advisable to compare the equivalent widths of the mapping line and other photospheric lines of the same species with the equivalent widths of a suitable comparison star. 

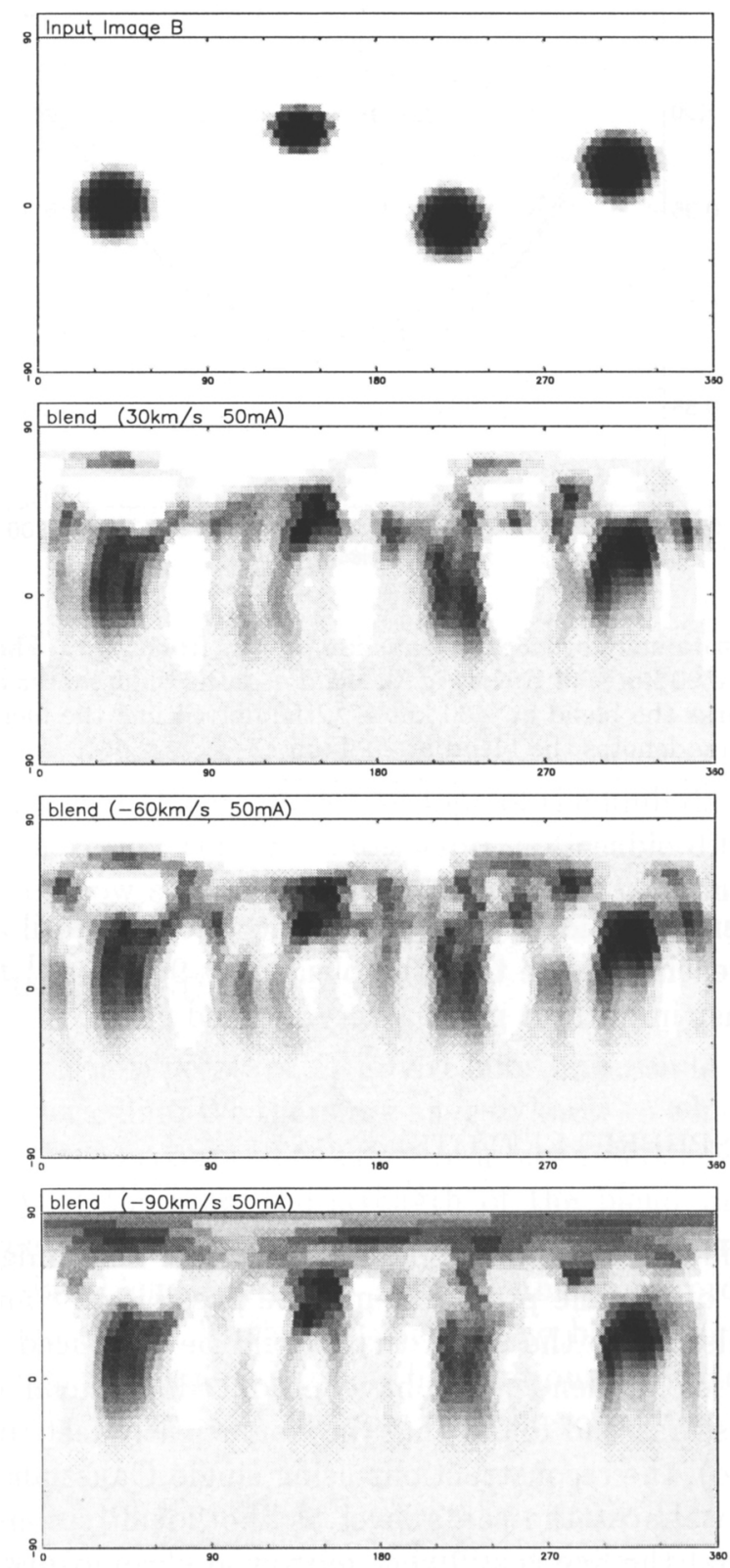

Figure 5. Artefacts due to the neglect of blends. The input image is shown in a). Fig. b) shows the reconstructed image from data produced with a blended Gaussian profile (blend at $-30 \mathrm{~km} \mathrm{~s}^{-1}$ ). The geocentric velocity had to be shifted to $-3.5 \mathrm{~km} \mathrm{~s}^{-1}$. c) As for b), but the blend is now at $-60 \mathrm{~km} \mathrm{~s}^{-1}$ and the geocentric velocity shift is $-5 \mathrm{~km} \mathrm{~s}^{-1}$ in order to achieve the best fit with a single Gaussian. For picture d) the smaller Gaussian is at $-90 \mathrm{~km} \mathrm{~s}^{-1}$ and the geocentric velocity shift is $-5.5 \mathrm{~km} \mathrm{~s}^{-1}$. 


\section{Discussion}

Doppler imaging is remarkably robust and mismatches that are 'symmetric' and affect both line wings to the same extent can be offset by adjusting the projected rotational velocity and the equivalent width. This then triggers the question on how to decide which parameters to choose for the final image.

All of the reconstructions tend to reproduce most of the features actually present in the original data. As the adopted parameters move away from their true values additional artefacts will appear. This suggests that the best parameters can be found by selecting the set of parameters for which the spot coverage is at a minimum for a given value of $\chi^{2}$. Alternatively, one could choose to use the image entropy instead of the spot coverage.

It is an advantage to make use of the vast number of photospheric lines in order to reconstruct the surface distribution on stars. Provided that blends are taken into account, reconstructions for several lines will increase the confidence in the final image. As different lines have different strengths and show different temperature and pressure dependencies, it is possible to constrain the range of values for the stellar parameters.

\section{Acknowledgements}

I would like to acknowledge the support from an IAU travel grant. I would also like to thank Andrew Collier Cameron and Jean-François Donati for useful discussions.

\section{References}

Collier Cameron A., Unruh Y. C. (1994) MNRAS, 269, 814

Collier Cameron A. (1992) in Byrne P. B., Mullan D. J., eds, Surface Inhomogeneities on Late-type Stars. Springer-Verlag, p. 33

Kürster M. (1993) . $A \& A, \mathbf{2 7 4}, 851$

Piskunov N. E., Tuominen I., Vilhu O. (1990) . $A \& A, \mathbf{2 3 0}, 363$

Piskunov N. E., Rice J. B. (1993) PASP, 105, 1415

Rice J. B., Wehlau W. H., Khokhlova V.L. (1989) . $A \& A, 208,179$

Unruh Y. C., Collier Cameron A. (1995) . MNRAS, 273, 1

Vogt S. S., Penrod G. D. (1983) . PASP, 95, 565

Vogt S. S., Penrod G. D., Hatzes A. P. (1987) . ApJ, 321, 496 


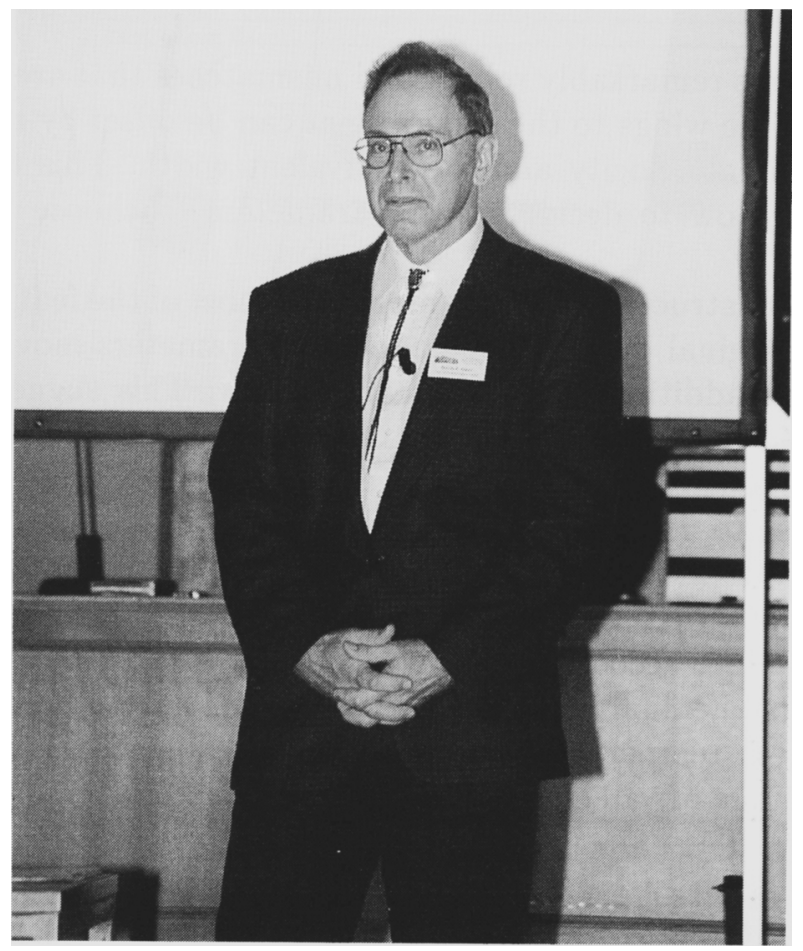

It's coffee and cookie break. The session chairman (David Gray, top) tries to get his flock back into the meeting hall. Obviously, not an easy task. (Bottom): J. Neff, P. Byrne, R. Dempsey, M. Güdel, F. Walter et al. still into heavy discussions.

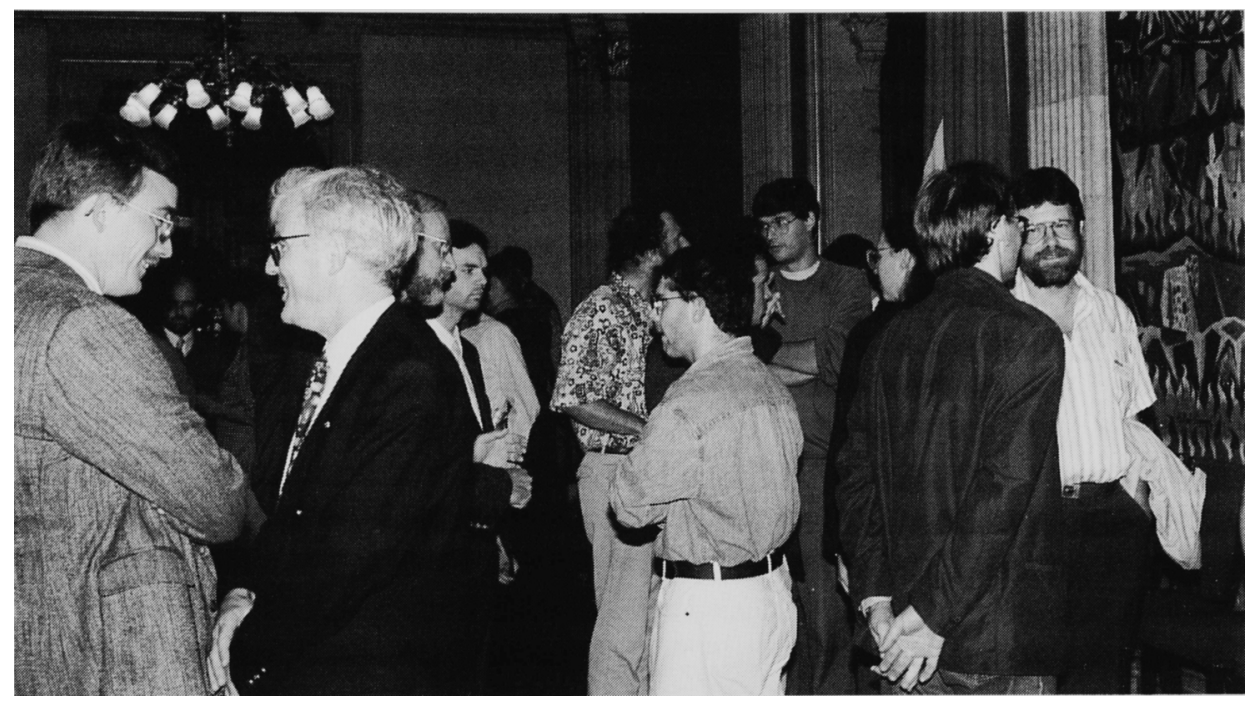

